\title{
Stretchable Sensors with Tunability and Single Stimuli- Responsiveness through Resistivity Switching Under Compressive Stress
}

Jarkko Tolvanen,* Joni Kilpijärvi, Olli Pitkänen, Jari Hannu, and Heli Jantunen

Cite This: ACS Appl. Mater. Interfaces 2020, 12, 14433-14442

Read Online

ACCESS | Lill Metrics \& More | 国 Article Recommendations ｜（s Supporting Information

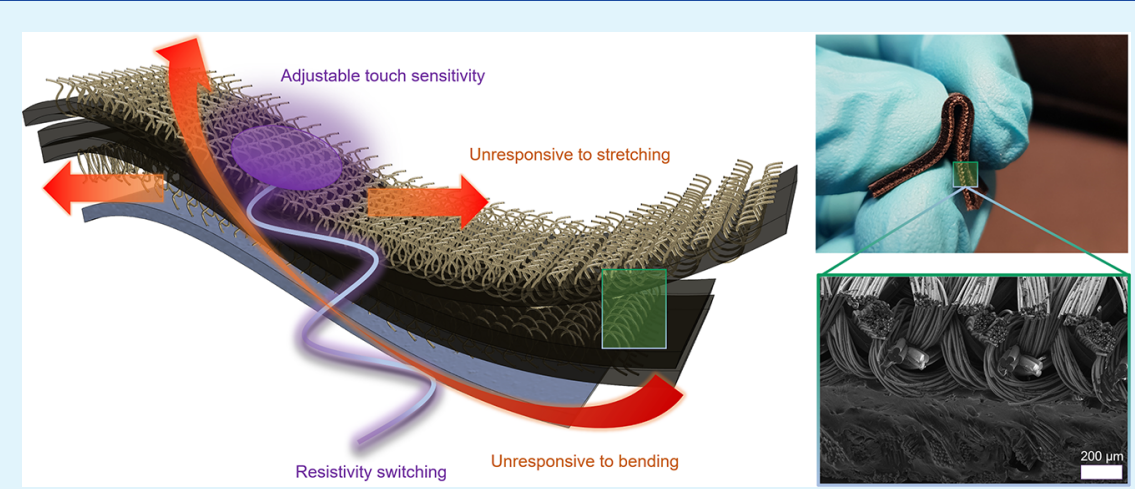

ABSTRACT: The fascinating human somatosensory system with its complex structure is composed of numerous sensory receptors possessing distinct responsiveness to stimuli. It is a continuous source of inspiration for tactile sensors that mimic its functions. However, to achieve single stimulus-responsiveness with mechanical decoupling is particularly challenging in the light of structural design and has not been fully addressed to date. Here we propose a novel structural design inspired by combining the characteristics of electronic skin (e-skin) and electronic textile (e-textile) into a hybrid interface to achieve a stretchable single stimuli-responsive tactile sensor. The stencil printable biocarbon composite/silver-plated nylon hybrid interface possesses an extraordinary resistance switching $\left(\Delta R / R_{0}\right.$ up to $\left.\sim 10^{4}\right)$ under compressive stress which is controllable by the composite film-thickness. It achieves a very high normal pressure sensitivity (up to $60.8 \mathrm{kPa}^{-1}$ ) in a wide dynamic range (up to $\sim 50 \mathrm{kPa}$ ) in the piezoresistive operation mode and can effectively decouple stresses induced by stretching or bending. In addition, the device is capable of high accuracy strain sensing in its capacitive operation mode through dimensional change dominant response. Because of these intriguing features, it has potential for the next-generation Internet of Things devices and user-interactive systems capable of providing visual feedback and more advanced robotics or even prosthetics.

KEYWORDS: biocarbon, composite, flexible electronics, insensitive, sensors, stimuli-responsive materials

\section{INTRODUCTION}

Mimicking the human somatosensory system has been the source of inspiration for tactile sensors having the capability of transducing physical and environmental stimuli into electrical signals. $^{1-5}$ Human skin has fascinating sensing capabilities through its numerous sensory receptors located on the top layers of the dermis and epidermis which have different rates of adaptability, receptive field sizes, and responsiveness to stimuli. ${ }^{4,6}$ The complex sensory systems enable precise physical interaction with the environment through the perception of touch, surface roughness, slippage, position, and motion. ${ }^{1,4,6}$ Even though surpassing the subtle sensing capabilities of human skin has proven to be a serious challenge, the efforts have paved the way for the next-generation of robotics, advanced prosthetics, healthcare, and medical devices. ${ }^{4-9}$
Recent research on mimicking the tactile sensation of human skin with capacitive, ${ }^{10-15}$ resistive, ${ }^{5,16-22}$ and other sensing mechanisms ${ }^{23-26}$ has focused on achieving a high sensitivity over the full transducer range of human skin, ${ }^{17,26-30}$ thus enabling the detection of normal and shear forces ${ }^{6,31}$ and even accurate measurement of a single-stimulus under dynamic deformations on curvilinear surfaces. ${ }^{7,17,31}$ Structural design is one of the most important aspects to achieve high performance for electronic skins (e-skins). Highly responsive e-skins have

Received: January 3, 2020

Accepted: March 2, 2020

Published: March 2, 2020 

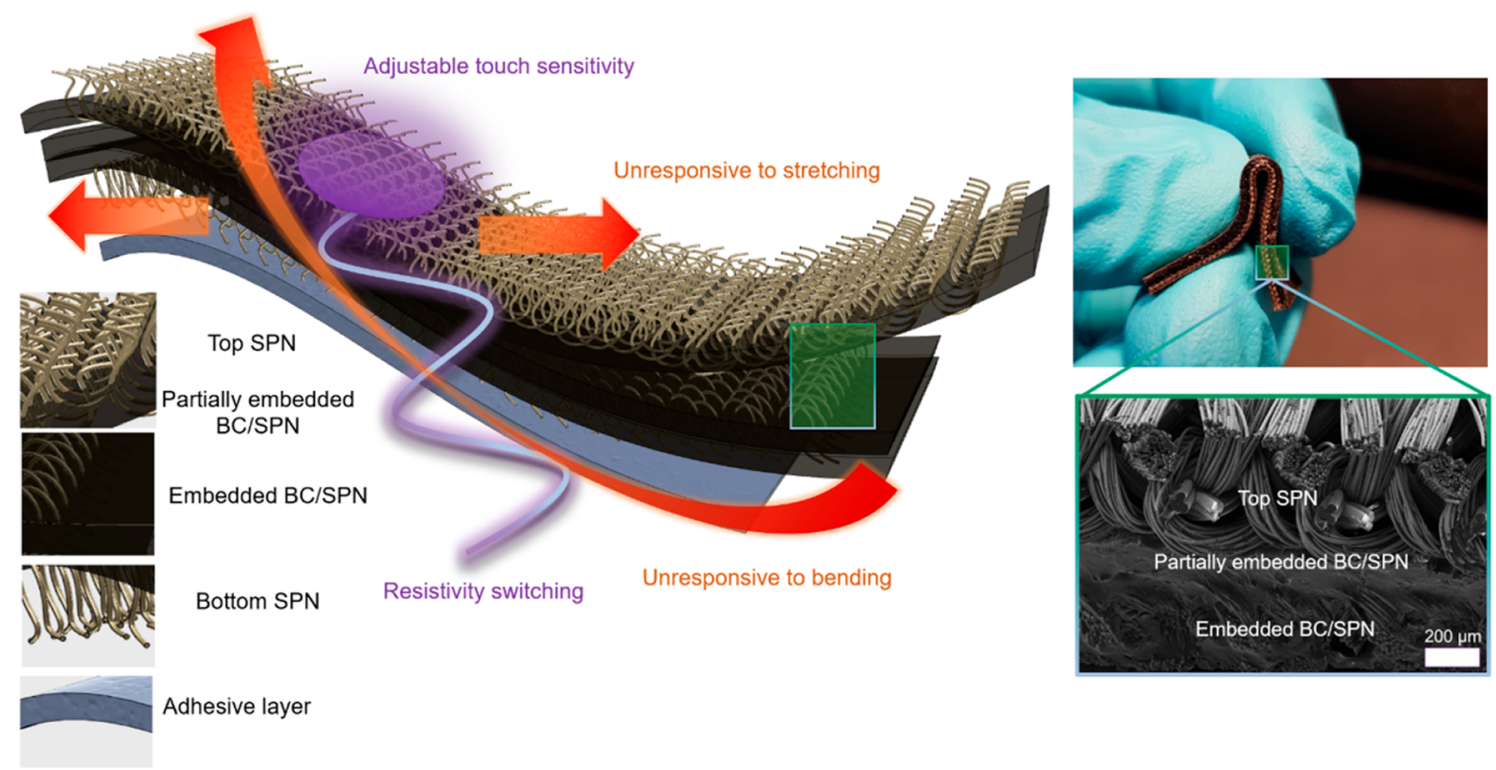

Figure 1. Schematic illustration, photograph, and FESEM image of a sensor consisting of two electrode layers made of silver-plated nylon (denoted as SPN), partially embedded and embedded biocarbon composite/silver-plated nylon interfaces (denoted as BC/SPN), and adhesive layer. The sensor shows resistance-switching with compressive stress but was found to be insensitive to stresses induced by stretching and bending in piezoresistive operation mode.

taken advantage of hierarchical structures inspired by nature, ${ }^{12,28,30}$ microstructured materials (such as micropyramids, microgrooves, microdomes), ${ }^{6,9,26,27,29}$ channels $^{17}$ and even hollow/porous ${ }^{32,33}$ structures, that allow control of the contact area and localized stress concentrations. ${ }^{29,34-36}$ Other efforts have focused on material development, such as creating supercapacitive iontronic sensing with an exceptionally high unit-area capacitance aiming toward excellent sensing capabilities. ${ }^{37,38}$ Yet there still exist only a few pressure sensors that can maintain a high sensitivity of $>50 \mathrm{kPa}^{-1}$ in the medium pressure regime (up to $100 \mathrm{kPa}$ ), thus capable of more closely mimicking the tactile sensing capabilities of natural skin. $5,23,26,31$

Structural design strategies for single stimuli-responsiveness and discrimination of other tactile inputs have not been fully explored to date. ${ }^{39}$ For instance, achieving accurate sensing of normal pressure under dynamic conditions on human skin has remained an issue for tactile sensors. ${ }^{39,40}$ All mechanical deformations change the outputs of the tactile sensor similarly due to the Poisson effect leading to poor decoupling of the inputs. $^{3,7,31,39}$ In this case, knowing the nature of the stimuli from the output can only be based on comparison and analysis of multiple time-dependent signal patterns to distinguish the different inputs. ${ }^{6,39}$ This leads to poor discrimination and the need for complex data processing due to signal interference. It is well-known that strains induced by bending decrease linearly with thickness. ${ }^{41}$ Even though such mechanics have been successfully utilized for nanoscale materials to design flexible electronics, it is not easily made use of in elastic materials and sensing layers or elements. ${ }^{7,41}$ Thus, it is essential to develop new strategies toward single stimuli-responsive sensors to avoid coupling and signal interference by the Poisson effect. To date, to the best of our knowledge, there does not exist any highly stretchable sensor that has an unusually high normal pressure sensitivity over a wide pressure regime, while simultaneously being insensitive to tensile and bending stress. $^{7,39,42-44}$
To address the challenge, we propose a novel structural design inspired by combining the characteristics of e-skin and electronic textile (e-textile) into a hybrid interface consisting of biocarbon composite/silver-plated nylon (denoted as BC/ SPN). Such seamless inclusions of e-skin and e-textiles have been mostly disregarded in the research community. Typically, soft elastomeric materials are only considered for encapsulation (i.e., waterproofing) or as glues for e-textiles, but not as part of the active structures. E-skins typically do not utilize textiles as substrates, electrodes, or as part of their active materials. However, hybrid approaches with ingenious designs have recently been proposed for sensors and have shown interesting properties in respect to many of their ordinary counterparts. $^{3,45,46}$

Herein, we demonstrate a new type of stretchable and highly sensitive sensor exhibiting insensitivity to the strains induced by stretching and bending. The device is capable of providing resistivity switching under compressive stress corresponding to a pressure sensitivity up to $60.8 \mathrm{kPa}^{-1}$ with a wide dynamic range (up to $\sim 50 \mathrm{kPa}$ ). The relative resistance change of the device is more than a thousandfold larger under normal pressure than during tensile or bending strain. More interestingly, we found the resistivity switching thresholds to be controllable by variation of the thickness of the stencil printed biocarbon film. The BC/SPN device is also capable, through its capacitive operation mode, of accurate strain sensing suitable for human motion monitoring. This work gives a detailed insight into the electromechanical properties of the device under capacitive and piezorestive operation modes. We have demonstrated its applicability to a user-interactive system with a 555-timer circuit able to instantaneously control the brightness of some LEDs relative to the applied force when touching the surface of a skin-attached device.

\section{RESULTS AND DISCUSSION}

Hybrid Structure. Figure 1 shows a schematic presentation of a BC/SPN device that comprises a multilayered hybrid 
a

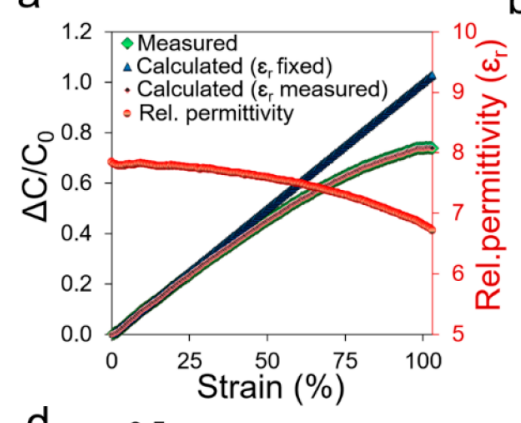

b

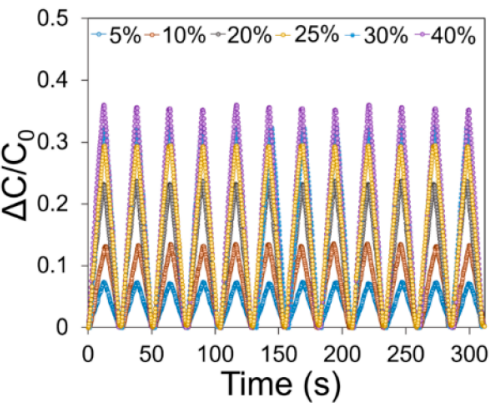

C

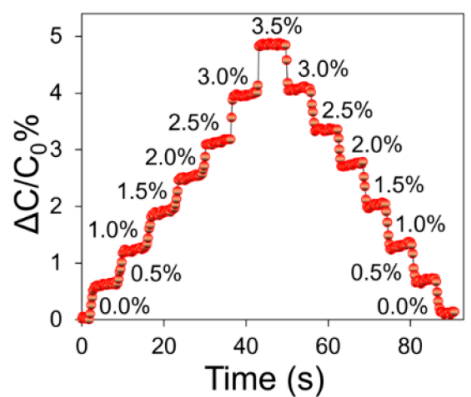

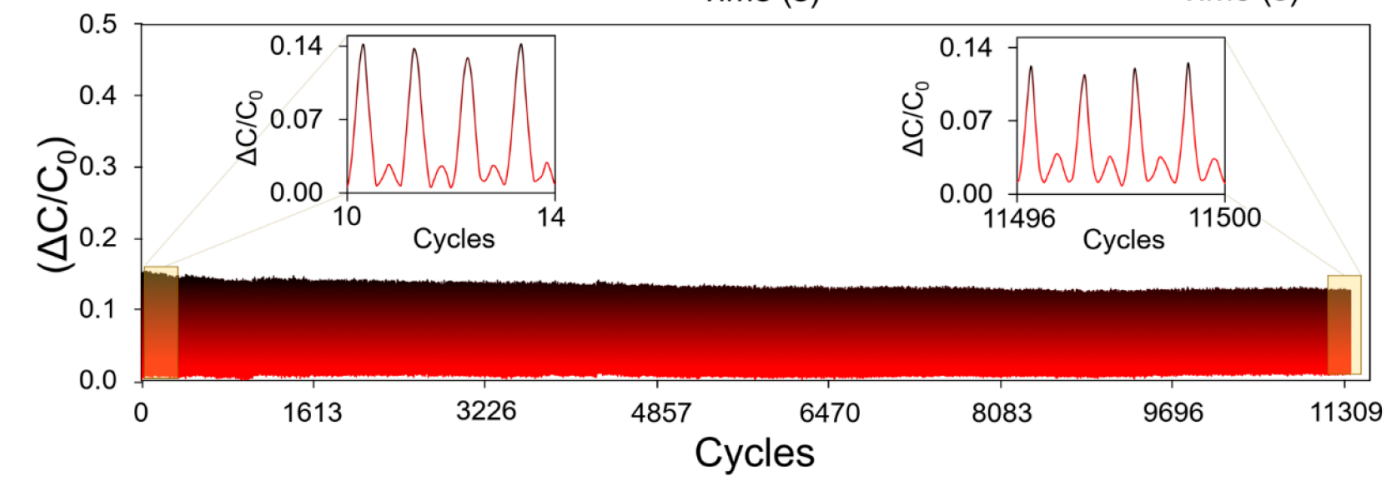

Figure 2. Characterization of capacitive response of BC/SPN device under tensile strain. (a) Relative capacitance change $\left(\Delta C / C_{0}\right)$ when applying strain up to $\sim 105 \%$, showing strain-dependence of relative permittivity $\left(\varepsilon_{\mathrm{r}}\right)$, calculated responses of the sensor (eq $S 1$ ) with fixed $\varepsilon_{\mathrm{r}}$ and $\varepsilon_{\mathrm{r}}-$ correction (strain rate $2.1 \% \mathrm{~s}^{-1}$ ). (b) $\Delta C / C_{0}$ when applying tensile strain of $5-40 \%$ (strain rates $0.42-3.33 \% \mathrm{~s}^{-1}$ ). (c) Step-and-hold test with $0.5 \% /$ step and $\sim 5 \mathrm{~s}$ holding period (strain rate $0.5 \% \mathrm{~s}^{-1}$ ). (d) Stability and durability test for $\sim 11500$ repetitions with combined cycles of stretching-releasing $(\varepsilon \approx 20 \%)$ and bending-releasing (bending angle $\sim 90^{\circ}$ or $\varepsilon \approx 6 \%$ ).

structure. The structure was inspired by combining e-skin (polymer-based smart materials) and e-textile (smart textiles) to realize a highly functional device. At a glance, the BC/SPN device resembles a typical parallel-plate capacitor structure having two overlapped electrodes in the form of silver-plated nylon (SPN) and a thin dielectric layer made of biocarbon composite (BC). The $\mathrm{BC} / \mathrm{SPN}$ enables high levels of deformability through its excellent stretchability but, more importantly, conformability as a wearable device (Figure S1 of the Supporting Information, SI). The BC/SPN was easily fabricated by stencil printing two layers of the composite solutions (urethane rubber/pyrolyzed pine $5 \mathrm{wt} \%$ ) onto polyimide taped SPN and applying pressure after stacking the top SPN onto a planar structure (SI Section 1.1 and Figure S2). The first BC film layer was almost completely embedded into the bottom SPN (Figure 1) while the second BC film layer can be only partially embedded into the top SPN, as shown in the FESEM images (Figures S3 and S4). This was partly due to the nature of knitted fabrics (i.e., nonsmooth surfaces), where empty spaces are formed within intermeshed and interconnected loops creating meandering paths. ${ }^{47}$ The applied pressure during printing controls the penetration depth of the stencil printed layers into the porous SPN. The embedded structure leads to stronger mechanical adhesion and layer-to-layer bonding between the films and the knitted fabrics, reducing the likelihood of delamination in the multilayered structure (SI Section 1.2 and Figure S5). A thinner dielectric composite layer resulted in a higher specific capacitance (i.e., capacitance per area) due to the reduced distance between the SPN strips, but more importantly it could adjust the resistance switching threshold under normal pressure (as illustrated in Figure 1). Also, the ingenious structural design of the device enabled insensitivity to other types of normal forces (tensile and bending stress). To get an insight into the electromechanical behavior for distinct deformations under normal stresses, the capacitive and piezoresistive operation modes of the BC/SPN were studied in detail.

Characteristics of Capacitive Operation. Stretchable sensors were characterized by their electrical responses under normal stress such as tensile strain. The strain sensitivity can be expressed as a gauge factor $\left(\mathrm{GF}=\left(\Delta C / C_{0}\right) / \varepsilon\right)$. For capacitive sensors it can be defined as a relative change of capacitance $\left(\Delta C / C_{0}\right)$ with respect to applied strain $(\varepsilon)$. The $\mathrm{BC} / \mathrm{SPN}$ devices displayed the typical sensitivity of a capacitive sensor $(\mathrm{GF} \approx 0.9$; Figure $2 \mathrm{a})$. The response of strain sensors with a parallel-plate capacitor structure are dictated by the Gauss's law defining the capacitance of an overlapped electrode structure (SI Section 1.3). Assumptions of Poisson's ratios ( $v=$ 0.5 for all materials) and relative permittivity being a fixed parameter would lead to a simplistic estimation of a capacitive sensor's response under stretching $\left(C=(1+\varepsilon) C_{0}\right)$. Therefore, linear responses would be expected as structural dimensional changes are the prime source of increased capacitance. However, the experimental results of $\mathrm{BC} / \mathrm{SPN}$ showed a very poor fit with the fixed relative permittivity $\left(\varepsilon_{\mathrm{r}}\right)$ value when the tensile strain increased above 50\% (Figure 2a).

The change of capacitance became increasingly nonlinear with increased strain, displaying a strong second-degree polynomial fit with negligible hysteresis (SI Section 1.4 and Figure S6). The fit suggests strain-dependent dielectric properties in the hybrid interface despite the low filler concentration of the biocarbon. The measured relative permittivity $\left(\varepsilon_{\mathrm{r}}\right)$ of $\mathrm{BC} / \mathrm{SPN}$ with embedded $\mathrm{BC} / \mathrm{SPN}$ interfaces decreased from 7.84 to 6.68 at a frequency of 1 $\mathrm{kHz}$ with applied tensile strain $(\varepsilon \approx 0-105 \%)$. The strain- 


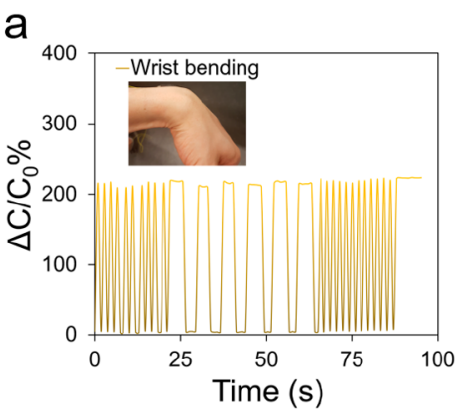

C

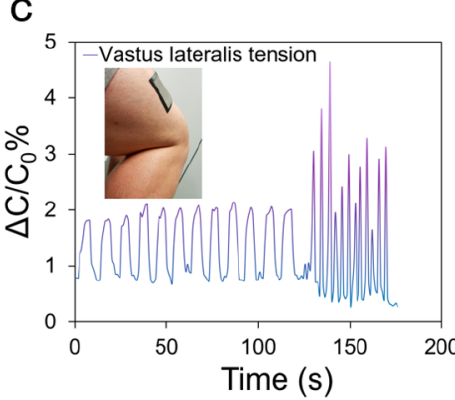

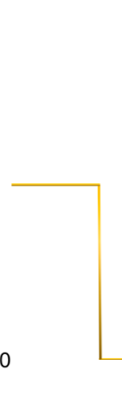

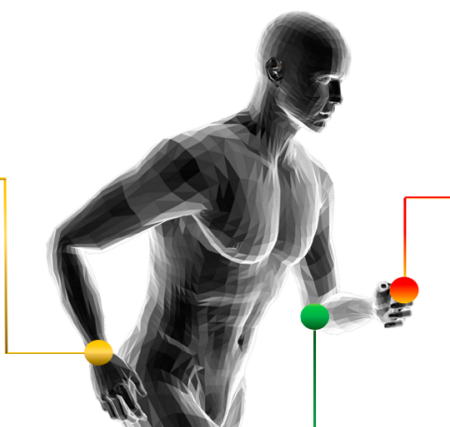

b

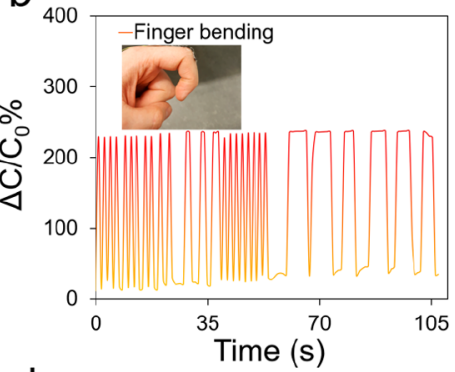

d

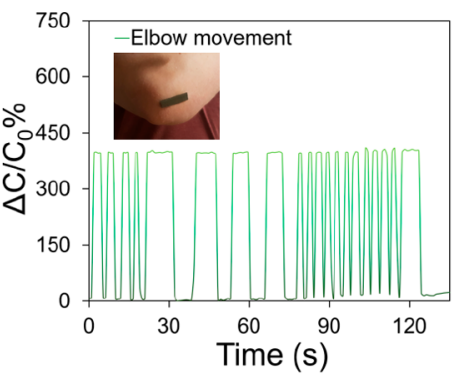

Figure 3. Application of BC/SPN device for human motion monitoring. (a) Response of BC/SPN when attached to a wrist and bending the wrist (angle $\sim 90^{\circ}$ ). (b) Response of $\mathrm{BC} / \mathrm{SPN}$ when attached to a forefinger and bending the finger (angle $\sim 90^{\circ}$ ). (c) Response of BC/SPN when attached to a vastus lateralis. Recording muscle tension during standing-sitting down motion and when raising one leg upward when standing still. (d) Response of BC/SPN when attached to elbow and swinging straightened arm toward the chest (angle $\sim 90^{\circ}$ ).

dependent dielectric properties at low frequencies have previously been found to partially relate to the straindependency of Maxwell-Wagner-Sillars polarization and charge dipoles, ${ }^{48}$ but more importantly it relates to the structural interfaces in the $\mathrm{BC} / \mathrm{SPN}$. We speculate that reorientation of the filler network in terms of increased horizontal separation and breakage of agglomerated carbon clusters within the polymer matrix negatively affects the relative permittivity $\left(\varepsilon_{\mathrm{r}}\right)$. Even so, the vertical connectivity of the filler network simultaneously would be increased by the Poisson effect. But also, increasing the porosity of the SPN decreased the relative permittivity further. Strain-dependent dielectric properties would require compensation to further improve a sensor's linearity which is important for the ondevice calibration process.

Figure $2 \mathrm{~b}$ shows the dynamic responses of a BC/SPN device when applying tensile strains of $5 \%, 10 \%, 20 \%, 25 \%, 30 \%$, and $40 \%$ with strain rates of $0.42-3.33 \% \mathrm{~s}^{-1}$. The BC/SPN displays increase of $\Delta C / C_{0}$ during repetitive stretchingreleasing cycles in accordance to Figure $2 \mathrm{a}$ when considering sample to sample variation. As expected, the capacitive response showed nontransient behavior with a uniform signal shape, indicating the reliability of the device for dynamic strain sensing. However, when applying a tensile strain of $\sim 25 \%$ with strain rates of $10-100 \% \mathrm{~s}^{-1}$, the devices showed strain rate dependencies in their $\Delta C / C_{0}$ (Figure S7). Even though the $\mathrm{BC} / \mathrm{SPN}$ network responds rapidly to stretching, it recovers insufficiently between stretching-releasing cycles due to viscoelasticity causing a time dependency. As previously reported, the strain rates in the range of $10-100 \% \mathrm{~s}^{-1}$ can also have a significant effect on the sensitivity of piezoresistive sensors. ${ }^{49}$ However, the capacitive response of BC/SPN under load is immutable under varied strain rates. This suggests that the relative permittivity of the BC/SPN with an embedded hybrid structure is immune to localized stress concentrations that could create volatile structural deformations in the presence of faster elastic responses.

Figure $2 \mathrm{c}$ shows the response of a BC/SPN device when applying a step-and-hold test with strain steps of $0.5 \%$ and $\sim 5 \mathrm{~s}$ hold period after each step. The response of the BC/SPN displayed small overshooting and undershooting behaviors related to the stress-relaxation resulting from the viscoelasticity of the polymer. When the applied strain increased to $3.5 \%$, the response became very stable indicating the reliability of the sensor for larger strain measurements. The limit of detection for the sensor were found to be $0.25 \%$ at a strain rate of $\sim 0.13 \% \mathrm{~s}^{-1}$ (Figure S8) corresponding to a tensile force of $\sim 0.38 \mathrm{~Pa}$. This indicates the devices' applicability even for low strain measurements despite stress-relaxation and the relatively small GF of the BC/SPN device. The low limit of detection can be accounted for by the low Young's modulus of the BC films and the small cross-sectional area of the sensor ascribed by Hooke's law (SI Section 1.5). The Young's modulus of BC films and the BC/SPN device in the linear stress-strain region were found to be approximately 0.25 and $0.46 \mathrm{MPa}$, respectively (Figures S5 and S9).

Stability and Durability of BC/SPN. Closely fitted stretchable electronic devices require electromechanical robustness to withstand the deformations caused by dynamic conditions. Plain unilateral movements caused by stretching could be considered inadequate for testing the robustness of wearable devices depending on their attachment on the body or skin. Therefore, a continuous multidirectional stress was applied to a BC/SPN device by stretching-bending-releasing movements for $\sim 39 \mathrm{~h}$ with a constant movement speed of 1 $\mathrm{mm} \mathrm{s}^{-1}$ (Figure 2d). The device's capacitive response was less sensitive to bending than to stretching, as indicated by the smaller $\Delta C / C_{0}$. This is partly accounted for by the relatively thin structure reducing the internal strain, as previously suggested. ${ }^{50}$ The $\mathrm{BC} / \mathrm{SPN}$ device showed an excellent cyclic 
stability and reliability with a negligible drift after $0.5 \mathrm{~h}$. We anticipate the stress-induced orientation of the filler-matrix network to be the reason for the drifting behavior during stretching-releasing cycles. Also, stress-relaxation of the materials of the $\mathrm{BC} / \mathrm{SPN}$ device could be a plausible cause for the evident change in the response. Altogether, the hybrid $\mathrm{BC} / \mathrm{SPN}$ structure displayed an excellent robustness to mechanical deformation despite the mechanical property transition between materials. The ingenious structure allowed a suppression of enfeebled points through the embedded BC/ SPN interfaces.

For stretchable electronics, an absence of machine washability can severely hinder their practicality for realworld applications. Typical failures during washing may occur after devices absorb water into their delicate structures or as result of mechanical wear during spin drying. These may lead to partial failures causing significant device instability or destructive failures where a device completely stops functioning. The durability of $\mathrm{BC} / \mathrm{SPNs}$ were tested during 30 machine washing cycles $\left(40{ }^{\circ} \mathrm{C}, 1200 \mathrm{rpm}\right.$, of $45 \mathrm{~min} /$ cycle $)$ when samples were put into a mesh bag and washed together with other clothes/garments (Figure S10). The mesh bag protects the SPNs from abrasive mechanical wear that may occur during spinning. This could lead to a harmful increase of the contact resistance. The $\Delta C / C_{0}$ of the $\mathrm{BC} / \mathrm{SNPs}$ (3 samples) were recorded before (Figure $\mathrm{S} 1 \mathrm{a}_{1-3}$ ) and after machinewashing cycles (Figure $\mathrm{S}_{10 \mathrm{~b}_{1-3}}$ ) with both dry and drip-dried samples, respectively. Also, the contact resistance variation was tested after each machine-washing cycle (after drip drying; Figure $\mathrm{S} 10 \mathrm{c}$ ). The $\mathrm{BC} / \mathrm{SPN}$ devices showed negligible variation of $\Delta C / C_{0}$ during repetitive stretching-releasing cycles before $\left(b_{1-3}\right)$ and after $\left(a_{1-3}\right)$ machine washing. More importantly, the average contact resistance of BC/SPNs were stable and structural dimensions were unchanged after 30 cycles indicating the high durability of the devices.

Strain Sensing with Capacitive Operation. To demonstrate the practicality of $\mathrm{BC} / \mathrm{SPN}$ for precise strain sensing, the devices were attached to skin with an adhesive layer to monitor human motion (Figure $3 a-d$ ). The daily movement of the human body can cause the skin to stretch by up to $\sim 50 \%$, which is well within the linearity region of a BC/ SPN device, where a change in relative permittivity has negligible effect on the $\Delta C / C_{0}$ (Figure 2a). As a result, the device showed repeatable output signals appearing and disappearing with motion and when returning to the initial position (i.e., straightening of wrist, and so on). More importantly, the device showed immutable signal amplitude with a constant motion range, a negligible static drift and unchangeable $\Delta C / C_{0}$ when the frequency of the motion increased. These together with no sign of overshooting and undershooting behavior indicated the high reliability of the device for precise human motion monitoring at larger strains. To monitor small strains caused by tension of the vastus lateralis, a sensor batch was fabricated with an increased size (effective area $\sim 25 \mathrm{~cm}^{2}$ ). Figure $3 \mathrm{c}$ shows the responses of the $\mathrm{BC} / \mathrm{SPN}$ when attached to the side of the leg to monitor muscle tension during standing up-sitting down motion (0$125 \mathrm{~s}$ ) and when standing still and raising the other leg to different heights $(125-175 \mathrm{~s})$. The $\Delta C / C_{0}$ increased or decreased as a result of the change in the motion. The expected overshooting and undershooting behavior for the $\mathrm{BC} / \mathrm{SPN}$ was observed at smaller strains.
The devices exhibited a relatively high specific capacitance $\left(>13 \mathrm{pF} \mathrm{cm}^{-2}\right.$ ) through the embedded BC/SPN interfaces. Thus, a high signal-to-noise ratio and noise immunity against environmental sources were achieved while having no effect on the reliability of the device. The achieved specific capacitances were up to 40 times higher than in other traditional nonsupercapacitive wearable sensors. ${ }^{7,36}$ The BC/SPN device has an ability to track motion and movements of the human body and even smaller strains caused by muscle tensions when in its high precision capacitive operation mode. The device holds great potential for accurate real time monitoring of typical human activities, either when directly attached to the skin or embedded into clothes and other textiles.

Piezoresistive and Capacitive Operation Modes under Normal Stresses. The piezoresistive and capacitive operation modes of a BC/SPN device were studied under normal stresses including compressive, tensile and bending stress. The BC/SPN showed a substantial film thickness dependent resistance switching behavior $\left(\Delta R / R_{0}\right.$ up to $\left.\sim 10^{4}\right)$ under compressive stress (Figure $4 \mathrm{a}$ ). The pressure sensitivity
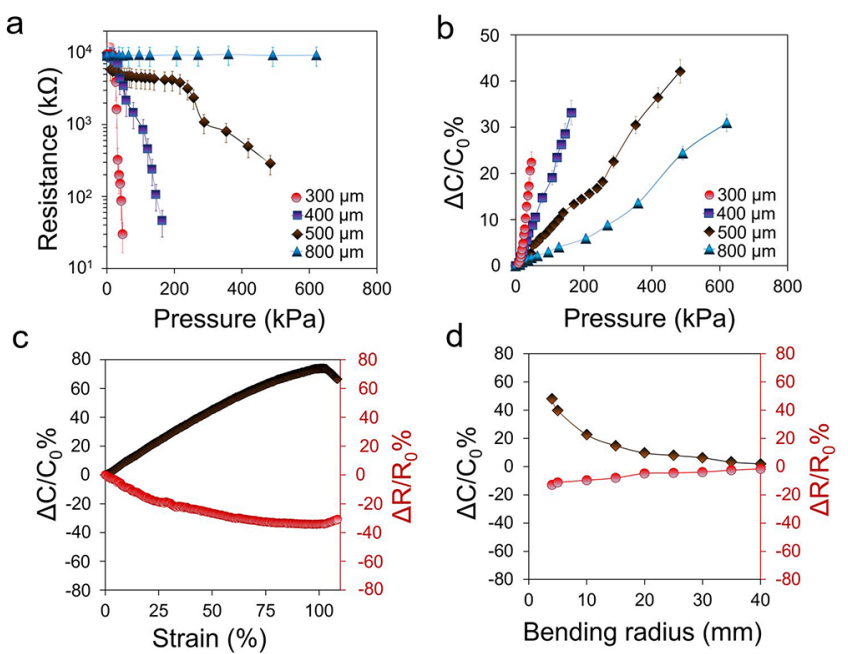

Figure 4. Characterization of piezoresistive and capacitive responses of $\mathrm{BC} / \mathrm{SPN}$ device. The resistance change (a) and relative capacitance change (b) under normal pressure with corresponding errors bars for films with thicknesses of $\sim 300-800 \mu \mathrm{m}$. Relative capacitance and resistance change when decreasing bending radius (c) and increasing tensile strain $(\mathrm{d})$.

can be expressed as the relative resistance change to applied pressure $\left(S=\left(\Delta R / R_{0}\right) / \Delta P\right)$. The resistance change of the $\mathrm{BC} / \mathrm{SPN}$ corresponded to remarkable maximum pressure sensitivities of $\sim 60.8 \mathrm{kPa}^{-1}$ in the pressure regime up to 50 $\mathrm{kPa}$ with prestress. The prestress required for sample attachment had a negative effect on the sensitivity as the initial resistance value of the $\mathrm{BC} / \mathrm{SPN}$ decreased from approximately $30-40 \mathrm{M} \Omega$ to $10 \mathrm{M} \Omega$. The response and recovery times of a sensor were less than $10 \mathrm{~ms}$ when applying normal pressure (SI Section 1.6 and Figure S11). It was found that the resistance switching thresholds shifted to a higher level of normal pressure when the film thickness increased. As a result, the average pressure sensitivity decreased to $\sim 0.05-$ $1.16 \mathrm{kPa}^{-1}$ (for film thickness $\sim 400-500 \mu \mathrm{m}$ ) in the high pressure regime $(>150 \mathrm{kPa})$. Interestingly, biocarbon composite films with thicknesses above $\sim 800 \mu \mathrm{m}$ tended to be unresponsive even to normal pressure with piezoresistive operation mode $(<1 \mathrm{MPa})$. The capacitive operation modes of 


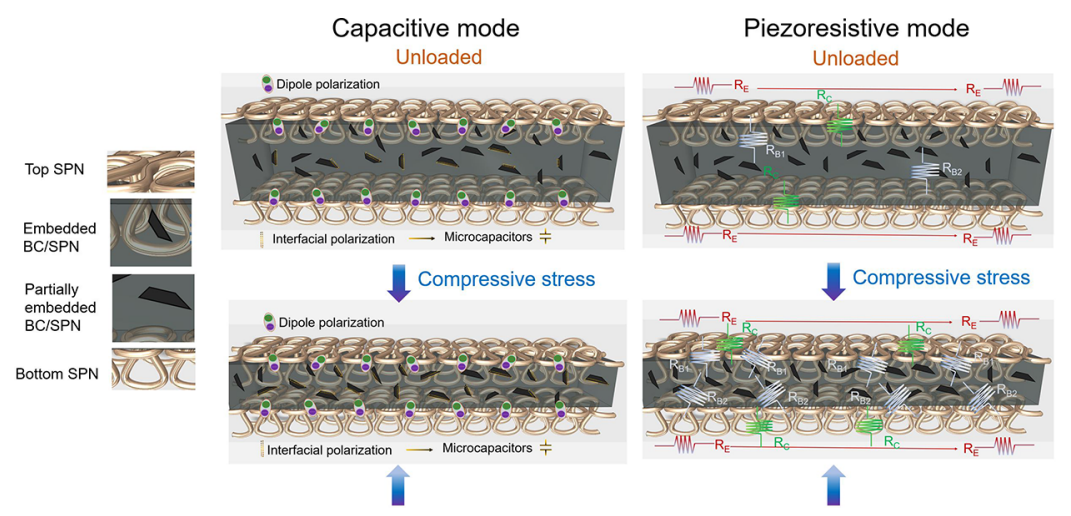

Figure 5. Schematic illustration of the capacitive and piezoresistive working principles of BC/SPN device under normal pressure.

the devices (SI Section 1.7) had altogether over a hundred times poorer pressure sensitivities (Figure 4b), but advantages such as near linear responsiveness (SI Section 1.8).

Tunable switching thresholds are important for diversification of the spectrum of applications, but they have not been easy to achieve. Zang et al. achieved tunability up to $100 \mathrm{kPa}$ by adjusting the modulus and/or dimensions of the suspended gate in a metal-oxide-semiconductor field-effect transistor (MOSFET). ${ }^{23}$ Bao et al. modulated the height of single-wall carbon nanotubes (SWCNTs) in microstructures to effectively control the switching thresholds from low to high pressure regime (up to $>100 \mathrm{kPa}$ ). ${ }^{5}$ Here we propose a simple strategy which is based on controlling the stencil printed biocarbon composite film thickness. Controlling the film thickness allows easily adjustable sensitivities and tunable resistance switching thresholds in a wide pressure regime $(>200 \mathrm{kPa})$.

Importantly, the piezoresistive operation mode of $\mathrm{BC} / \mathrm{SPN}$ showed strong responsiveness only toward compressive stress, while it was unresponsive to applied tensile and bending stresses. The BC/SPN relative resistance changes $\left(\Delta R / R_{0}\right)$ varied by only by $-12 \%$ and $-35 \%$ when the bending radius $\left(r_{\mathrm{b}}\right)$ decreased to $4 \mathrm{~mm}$ (Figure $4 \mathrm{c}$ ) and applied tensile strain increased to $\sim 100 \%$ (Figure $4 \mathrm{~d}$ ), respectively. Previous bending-insensitive sensors have shown substantial pressure sensitivities only in narrow pressure regimes $(\leq 1 \mathrm{kPa})$ in comparison to this work (Table $S 1$ ). The highly responsive nature of the hybrid interface allowed more than a thousandfold larger relative resistance change under normal pressure than under tensile or bending strain in the demonstrated operational ranges (for a film with thickness $\sim 300 \mu \mathrm{m}$ ). Decoupling Poisson's effect is not possible with capacitive operation mode as the device reacts in similar manner toward any mechanical stimuli (Figure $4 \mathrm{~b}-\mathrm{d}$ and Table S2).

Achieving pressure sensors that are insensitive to strain and bending is particularly important when they are directly attached on soft, curvilinear, and movable surfaces such as human skin. The natural movement of the skin during our daily activities leads to the development of stresses associated with mechanical deformations that can significantly impair the accuracy of an ordinary sensor. To demonstrate efficient decoupling for measuring normal pressures, negligible small differences between flat, bended and stretched states must be shown. Figure S12 shows that in piezoresistive operation mode the device is able to record changes in normal pressure even when attached to a curvilinear surface $\left(r_{\mathrm{b}}=4 \mathrm{~mm}\right)$ or being stretched $(\varepsilon=100 \%)$. Only the initial resistance values of the device slightly decrease when bended or stretched (Figure $4 c, d)$ in comparison to being flat.

The working principles of $\mathrm{BC} / \mathrm{SPN}$ device with capacitive and piezoresistive operation modes (eqs S1, S3, and S4) are illustrated in Figure 5. The capacitive change of the device is controlled by the dimensional changes occurring under normal stresses (eqs S1 and S3). The relative permittivity changes moderately as a result of deformation leading to nonlinear responses (Figures $2 \mathrm{a}$ and $4 \mathrm{~b}$ ). Structural compression leads to formation of more densely packed microscopic capacitor network (i.e., separation of filler particles and SPN decrease) and interfacial polarization can increase. Due to nature of the filler and its low wt \% in the composite, there are no substantial changes in the capacitance under normal pressure. The affinity of the piezoresistive operation mode under tensile and bending stress relates to the parallel-plate capacitor type of structure leading to dimensional change dominant responses (SI Section 1.9).

However, for normal pressure, the resistivity change of the device is dominated by the contact resistance change and the bulk resistivity change of the embedded BC/SPN layers forming a parallel resistance. The average resistivity of the biocarbon filler was found to be $\sim 660 \Omega \cdot \mathrm{m}$, and the biocarbon composite could be considered as an insulator (Table S3). We speculate that the low conductive filler plays a major role in controlling the resistivity under deformation of the SPN. At the initial stage, the total resistance of the device can be expressed as $R=R_{\mathrm{E}}+\left[1 / R_{\mathrm{C}}+1 /\left(R_{\mathrm{B} 1}+R_{\mathrm{B} 2}\right)\right]$, where $R_{\mathrm{E}}, R_{\mathrm{C}}, R_{\mathrm{B} 1}$, and $R_{\mathrm{B} 2}$ denote the resistance of electrodes, contact resistance to SPN and the resistance of the embedded/partially embedded $\mathrm{BC} / \mathrm{SPN}$ layers, respectively. The device has a high resistance when unloaded due to a high parallel resistance in the presence of the small number of contact junctions and as the resistances $R_{\mathrm{C}}, R_{\mathrm{B} 1}$, and $R_{\mathrm{B} 2}$ are large.

Under compressive stress, the number of conductive junctions (denoted as $n$ ) significantly increases in the SPN/ filler network having a relatively large particle size $(\sim 20 \mu \mathrm{m})$. Thus, the resistance of the BC/SPN becomes $R=R_{\mathrm{E}}+\left(1 / R_{\mathrm{C}}\right.$ $\left.+1 /\left[n\left(R_{\mathrm{B} 1}+R_{\mathrm{B} 2}\right)\right]\right)$. The porosity of the intermeshed and interconnect structure of SPN reduces as a function of normal pressure leading to an increased contact area and decreased contact resistance within hybrid interfaces. These lead to an abrupt decrease in the parallel resistance under normal pressure giving rise to a giant resistivity switching at an adequate load. Furthermore, we speculate that the complex stress dependent conduction of biocarbon contributes to the 
a

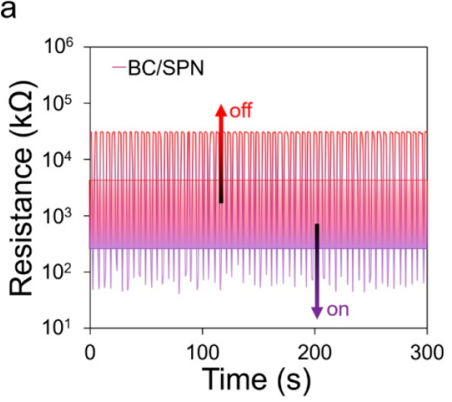

b
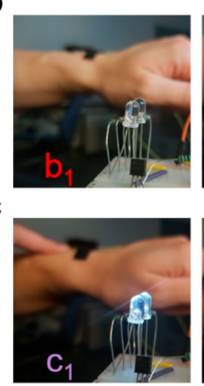
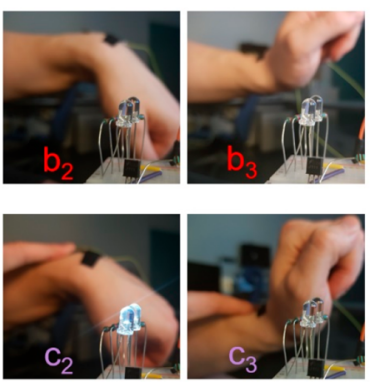

Figure 6. Pressure switching behavior in BC/SPN device. (a) BC/SPN resistance changes by three-orders of magnitude when applying a pressure of $\sim 50 \mathrm{kPa}$. LED circuit is controllable by applying a pressure to the skin-attached BC/SPN. (b) Without applied pressure to BC/SPN the LEDs are off $\left(b_{1}\right)$ despite the downward $\left(b_{2}\right)$ and upward movement of wrist $\left(b_{3}\right) .(c)$ LEDs switch on as pressure is applied ( $\left.c_{1}-c_{2}\right)$, while LEDs switch off during upward bending motion $\left(c_{3}\right)$.

decrease of resistance of the conductive junctions within hybrid interfaces. ${ }^{51-54}$

The controllability over the resistance switching thresholds with film thickness was found to be related to the higher degree of compressibility at the hybrid interface than at the biocarbon composite film under normal pressure due to Poisson's ratios. For deformations associated with tensile and bending stress the parallel resistance remains large even though the number of contact junctions increase as a result of the Poisson's effect. There is only small contact area change and contact resistance remains larger as the porosity of SPN increases (i.e., dimensional changes dominate).

Application as Touch Sensitive Device. Figure 6a shows the pressure switching characteristics of a BC/SPN device (film thickness $\sim 300 \mu \mathrm{m}$ ) during repetitive press-release cycles $(\sim 50 \mathrm{kPa})$. The $\mathrm{BC} / \mathrm{SPN}$ showed highly reversible resistance switching behavior, where the resistance decreased by 3 orders of magnitude from an initial resistance value of $\sim 30 \mathrm{M} \Omega$ to $\sim 30 \mathrm{k} \Omega$ as external pressure was applied. To further demonstrate the resistance-switching characteristic of $\mathrm{BC} /$ $\mathrm{SPN}$, a 555-timer circuit with a driving voltage of $12 \mathrm{~V}$ was designed. The "off" and "on"-states in the circuit corresponded to the high and low resistance states of the BC/SPN (Figure $6 \mathrm{a}-\mathrm{c}) . \mathrm{BC} / \mathrm{SPN}$ were attached to a wrist with an adhesive layer to demonstrate the resistive switching function for wearable devices. Typically, a gentle human touch can generate a pressure up to $\sim 10 \mathrm{kPa}$. As a result, the light emitting diodes (LEDs) could be instantaneously switched on/off by simply touching the surface of the device with a finger (Figure $6 b, c$ ). After applying a force to the device, the response time to change the intensity of the LEDs was less than $10 \mathrm{~ms}$. The resistance-switching characteristic showed the expected insensitivity to stretching/bending caused by wrist movements $\left(b_{1}-b_{3}\right)$. However, touching the surface of the BC/SPN and bending the wrist upward turned the LEDs off $\left(c_{1}-c_{3}\right)$. Such motion decreased the localized compressive stress below the resistance-switching threshold leading to increased resistivity in the overlapped device structure. Moreover, the brightness of the LEDs could be easily adjustable by controlling the applied pressure (i.e., tuning the resistance of the device) as shown in the Video S1.

Ultrasensitive mechanoresponsive materials (MRM) with switching characteristics are urgently needed in low-power consumption user-interactive systems. User-interactive systems integrated with other electronic components (such as electrochromic devices) are capable of providing visual-feedback of the sensors' electrical responses. Such visuality allows the electrical responses of devices to become readable even to the human eye. The ultrahigh resistance or current-switching on/ off ratios in MRM can enable lower driving voltages (i.e., lower power consumption) for the user-interactive systems without using additional electronic components (such as amplifier circuits). The performance characteristics of BC/SPN were compared to previously developed MRM displaying switching phenomena (Table S4). The previously developed switching devices can ultimately be classifiable into two categories: (i) flexible pressure switching devices with small working ranges and (ii) stretchable strain switching devices. However, a few stretchable pressure switching devices distinct from these two categories have been demonstrated which are similar to this work. Chen et al. fabricated a stretchable cloth-based piezoresistive structure with an $I_{\mathrm{ON}} / I_{\mathrm{OFF}}$ ratio of $\sim 10^{7}$ having a workable pressure range up to $<3 \mathrm{kPa}$. ${ }^{46}$ The cloth-based devices demonstrated an extremely low working voltage of 0.1 $\mathrm{V}$, but a major drawback to be considered was the lack of tunable sensitivities and current switching thresholds, as in most other devices (Table S4). Bao et al. fabricated CNT/ PDMS composites with interlocked microdome arrays presenting an $R_{\mathrm{ON}} / R_{\mathrm{OFF}}$ ratio of $\sim 10^{6}$ with highly tunable sensitivities and resistance switching thresholds with a wide range $(\sim 10-700 \mathrm{kPa})^{5}$. Notwithstanding their excellence, considerable challenges of many micropatterned materials relate to their fabrication processes which are cumbersome, costly, and have scalability issues. The stencil printing of BC/ SPN offers a simple and low-cost fabrication method that can support large-scale manufacturing and prototyping.

\section{CONCLUSIONS}

In summary, we have presented a novel structural design inspired by combining the characteristics of e-skin and e-textile into a stretchable single stimuli-responsive sensor. The lowcost fabrication method of stencil printing biocarbon composite films onto silver-plated nylon enables control of the resistivity switching under compressive stress through variation of the film thickness. The piezoresistive operation mode of the devices enabled a remarkable pressure sensitivity (up to $\left.60.8 \mathrm{kPa}^{-1}\right)$ with a wide dynamic range $(\sim 50 \mathrm{kPa})$. This corresponded to a maximum relative resistance change of $\Delta R$ / $R_{0} \sim 10^{4}$ from the low to medium pressure regime, while showing unresponsiveness to tensile strain $\left(\Delta R / R_{0} \approx-30 \%\right.$ for $\varepsilon>100 \%)$ and bending $\left(\Delta R / R_{0} \approx-12 \%\right.$ for $\left.\mathrm{r}_{\mathrm{b}}=4 \mathrm{~mm}\right)$. Decoupling of strain was not possible with capacitive operation mode due to dimensional change dominant responses. However, the capacitive operation mode of the device enabled 
high precision strain sensing for human motion monitoring. The high stability of the device was illustrated by $39 \mathrm{~h}$ of continuous operation. Excellent durability was demonstrated by machine washing for 30 cycles at $40{ }^{\circ} \mathrm{C}$ with $1200 \mathrm{rpm}$ with no changes in physical dimensions, electromechanical responses or contact resistance of the electrodes. The response and recovery times of a sensor were less than $10 \mathrm{~ms}$ for normal pressure. The fascinating characteristics of these devices are anticipated to pave the way for new user-interactive systems and environments capable of visual feedback and interactions with human, robotics, or even prosthetics.

\section{METHODS}

Preparation of BC/SPN Sensors Structure. Urethane rubber (Vytaflex 20, Smooth-On) A-component was mixed at room temperature with 5 wt $\%$ of crushed pine powder (pyrolized at 370 ${ }^{\circ} \mathrm{C}$, particle size $\sim 20 \mu \mathrm{m}$, Noireco Oy). The solution was repeatedly vacuum degassed before and after adding the B-component (mixing ratio $1 \mathrm{~A}: 1 \mathrm{~B}$ ). Then the poured urethane/biochar $5 \mathrm{wt} \%$ solution was stencil printed onto polyimide film taped (Kapton, $3 \mathrm{M}$ ) silver-plated nylon fabric (P130, MedTex, Kitronik) to form a composite film/ conductive fabric layer with a thickness of $\sim 500-850 \mu \mathrm{m}$. The layered structure was cured at $23{ }^{\circ} \mathrm{C}$ for $16-24 \mathrm{~h}$. Similarly, another composite film layer was stencil printed on top of the cured layered structure to adhere the second fabric to the existing layers. Then, the multilayered composite film/textile structure with a total thickness of $\sim 950-1700 \mu \mathrm{m}$ was cured at $23{ }^{\circ} \mathrm{C}$ for $16-24 \mathrm{~h}$ and postcured at 65 ${ }^{\circ} \mathrm{C}$ for $8 \mathrm{~h}$. The skin-adhesive layer of silicone was fabricated as previously reported. ${ }^{3}$

Material Characterization. Field emission scanning electron microscopy (FESEM, Zeiss Ultra Plus) was used to study the microstructures of the samples.

Electrical and Mechanical Measurements. The stress-strain curves of the materials and developed sensors were recorded with a Linkam TST350 tensile stress testing system with a $200 \mathrm{~N}$ force transducer at $23{ }^{\circ} \mathrm{C}$.

Electromechanical Measurement. Electromechanical responses of the sensors were recorded with a LabVIEW controlled Agilent A4284A Precision LCR-meter with an amplitude of $1 \mathrm{~V}$ at a frequency of $1 \mathrm{kHz}$ while the samples were attached to a moving stage (Linkam TST350 with $200 \mathrm{~N}$ force transducer) and a vertical sample holder, where compressive stress and/or movement were applied via a computer controllable piston (Festo). Due to the vertical attachment of the samples when applying pressure, prestress was used $(<5 \mathrm{kPa})$. The applied force of the piston was calculated through a dynamic load cell (Omega Engineering, INC DLC 101-100) placed on the opposite side of piston while measuring the peak-to-peak voltage with an MSO-X-3054A oscilloscope. The electromechanical responses were recorded at $23{ }^{\circ} \mathrm{C}$.

\section{ASSOCIATED CONTENT}

\section{SI Supporting Information}

The Supporting Information is available free of charge at https://pubs.acs.org/doi/10.1021/acsami.0c00023.

Photographs of a sensor; illustration of the fabrication process; FESEM images of sensor structure; FESEM images of sensor under stress; stress-strain curves for sensors with two configurations; capacitive and piezoresistive responses when applying and releasing tensile strain; capacitive response with varied strain rate; capacitive response under small strain (0.25-0.5\%); stress-strain curves for biocarbon composite films; durability testing for sensor during machine washing (30 cycles); response and recovery times of a sensor under normal pressure; piezoresistive responses of a sensor when flat, bent, or stretched; comparison of bending-insensitive sensors; comparison of the two operation modes; electrical properties of biocarbon and composite; and comparison of resistive and capacitive switching devices (PDF)

Movie S1, resistive switching demonstration with LEDs (MP4)

\section{AUTHOR INFORMATION}

\section{Corresponding Author}

Jarkko Tolvanen - Microelectronics Research Unit, Faculty of Information Technology and Electrical Engineering, University of Oulu, Oulu FIN-90014, Finland; 10 orcid.org/0000-00024960-2286; Email: jarkko.tolvanen@oulu.fi

\section{Authors}

Joni Kilpijärvi - Microelectronics Research Unit, Faculty of Information Technology and Electrical Engineering, University of Oulu, Oulu FIN-90014, Finland

Olli Pitkänen - Microelectronics Research Unit, Faculty of Information Technology and Electrical Engineering, University of Oulu, Oulu FIN-90014, Finland; O orcid.org/0000-00032870-3229

Jari Hannu - Microelectronics Research Unit, Faculty of Information Technology and Electrical Engineering, University of Oulu, Oulu FIN-90014, Finland

Heli Jantunen - Microelectronics Research Unit, Faculty of Information Technology and Electrical Engineering, University of Oulu, Oulu FIN-90014, Finland

Complete contact information is available at: https://pubs.acs.org/10.1021/acsami.0c00023

\section{Author Contributions}

J.T. designed and performed electromechanical characterizations. J.K. designed the LabVIEW program. O.P. analyzed the microstructures. All authors contributed to the final version of the article.

\section{Notes}

The authors declare no competing financial interest.

\section{ACKNOWLEDGMENTS}

This research was financially supported by the projects Grelectronics (1569/31/2017, Business Finland) and ENTITY (Infotech Oulu, University of Oulu). This work was supported by the Academy of Finland Research Infrastructure "Printed Intelligence Infrastructure (PII-FIRI, grant no. 320017). Noireco $\mathrm{Oy}$ is acknowledged for preparing and providing the pyrolized pine. Florian Ouvrard is acknowledged for the design and fabrication of the 555-timer circuit for the light emitting diode switching demonstration.

\section{REFERENCES}

(1) Hua, Q.; Sun, J.; Liu, H.; Bao, R.; Yu, R.; Zhai, J.; Pan, C.; Wang, Z. L. Skin-Inspired Highly Stretchable and Conformable Matrix Networks for Multifunctional Sensing. Nat. Commun. 2018, 9 (1), 111.

(2) Guo, R.; Chen, J.; Yang, B.; Liu, L.; Su, L.; Shen, B.; Yan, X. InPlane Micro-Supercapacitors for an Integrated Device on One Piece of Paper. Adv. Funct. Mater. 2017, 27 (43), 1-11.

(3) Tolvanen, J.; Hannu, J.; Jantunen, H. Stretchable and Washable Strain Sensor Based on Cracking Structure for Human Motion Monitoring. Sci. Rep. 2018 DOI: 10.1038/s41598-018-31628-7.

(4) Chortos, A.; Liu, J.; Bao, Z. Pursuing Prosthetic Electronic Skin. Nat. Mater. 2016, 15 (9), 937-950. 
(5) Chou, H. H.; Nguyen, A.; Chortos, A.; To, J. W. F.; Lu, C.; Mei, J.; Kurosawa, T.; Bae, W. G.; Tok, J. B. H.; Bao, Z. A ChameleonInspired Stretchable Electronic Skin with Interactive Colour Changing Controlled by Tactile Sensing. Nat. Commun. 2015, 6, 1-10.

(6) Boutry, C. M.; Negre, M.; Jorda, M.; Vardoulis, O.; Chortos, A.; Khatib, O.; Bao, Z. A Hierarchically Patterned, Bioinspired e-Skin Able to Detect the Direction of Applied Pressure for Robotics. Sci. Robot. 2018, 3(24), eaau6914.

(7) Lee, S.; Reuveny, A.; Reeder, J.; Lee, S.; Jin, H.; Liu, Q.; Yokota, T.; Sekitani, T.; Isoyama, T.; Abe, Y.; Suo, Z.; Someya, T. A Transparent Bending-Insensitive Pressure Sensor. Nat. Nanotechnol. 2016, 11, 472.

(8) Pan, L.; Chortos, A.; Yu, G.; Wang, Y.; Isaacson, S.; Allen, R.; Shi, Y.; Dauskardt, R.; Bao, Z. An Ultra-Sensitive Resistive Pressure Sensor Based on Hollow-Sphere Microstructure Induced Elasticity in Conducting Polymer Film. Nat. Commun. 2014 DOI: 10.1038/ ncomms4002.

(9) Qiu, Z.; Wan, Y.; Zhou, W.; Yang, J.; Yang, J.; Huang, J.; Zhang, J.; Liu, Q.; Huang, S.; Bai, N.; Wu, Z.; Hong, W.; Wang, H.; Guo, C. F. Ionic Skin with Biomimetic Dielectric Layer Templated from Calathea Zebrine Leaf. Adv. Funct. Mater. 2018, 28 (37), 1-9.

(10) Li, R.; Si, Y.; Zhu, Z.; Guo, Y.; Zhang, Y.; Pan, N.; Sun, G.; Pan, T. Supercapacitive Iontronic Nanofabric Sensing. Adv. Mater. 2017, 29 (36), 1-8.

(11) Li, S.; Pan, N.; Zhu, Z.; Li, R.; Li, B.; Chu, J.; Li, G.; Chang, Y.; Pan, T. All-in-One Iontronic Sensing Paper. Adv. Funct. Mater. 2019, 29 (11), 1-11.

(12) Wan, Y.; Qiu, Z.; Huang, J.; Yang, J.; Wang, Q.; Lu, P.; Yang, J.; Zhang, J.; Huang, S.; Wu, Z.; Guo, C. F. Natural Plant Materials as Dielectric Layer for Highly Sensitive Flexible Electronic Skin. Small 2018, 14 (35), 1801657.

(13) Ho, D. H.; Cheon, S.; Hong, P.; Park, J. H.; Suk, J. W.; Kim, D. H.; Han, J. T.; Cho, J. H. Multifunctional Smart Textronics with Blow-Spun Nonwoven Fabrics. Adv. Funct. Mater. 2019, 29 (24), 1-9.

(14) Gerratt, A. P.; Michaud, H. O.; Lacour, S. P. Elastomeric Electronic Skin for Prosthetic Tactile Sensation. Adv. Funct. Mater. 2015, 25 (15), 2287-2295.

(15) Shi, H.; Al-Rubaiai, M.; Holbrook, C. M.; Miao, J.; Pinto, T.; Wang, C.; Tan, X. Screen-Printed Soft Capacitive Sensors for Spatial Mapping of Both Positive and Negative Pressures. Adv. Funct. Mater. 2019, 29 (23), 1-10.

(16) Lai, Y.; Ye, B.; Lu, C.; Chen, C.; Jao, M.; Su, W.; Hung, W.; Lin, T.; Chen, Y. Extraordinarily Sensitive and Low-Voltage Operational Cloth-Based Electronic Skin for Wearable Sensing and Multifunctional Integration Uses: A Tactile-Induced Insulating-toConducting Transition. 2016, 1286-1295 DOI: 10.1002/ adfm.201503606.

(17) Liu, W.; Liu, N.; Yue, Y.; Rao, J.; Cheng, F.; Su, J.; Liu, Z.; Gao, Y. Piezoresistive Pressure Sensor Based on Synergistical Innerconnect Polyvinyl Alcohol Nanowires/Wrinkled Graphene Film. Small 2018. 14151704149.

(18) Li, W.; Guo, J.; Fan, D. 3D Graphite-Polymer Flexible Strain Sensors with Ultrasensitivity and Durability for Real-Time Human Vital Sign Monitoring and Musical Instrument Education. Adv. Mater. Technol. 2017, 2 (6), 1-9.

(19) Jeong, Y. R.; Park, H.; Jin, S. W.; Hong, S. Y.; Lee, S. S.; Ha, J. S. Highly Stretchable and Sensitive Strain Sensors Using Fragmentized Graphene Foam. Adv. Funct. Mater. 2015, 25 (27), 4228-4236.

(20) Sheng, L.; Liang, Y.; Jiang, L.; Wang, Q.; Wei, T.; Qu, L.; Fan, Z. Bubble-Decorated Honeycomb-Like Graphene Film as Ultrahigh Sensitivity Pressure Sensors. Adv. Funct. Mater. 2015, 25 (41), 65456551.

(21) Liu, X.; Liu, D.; Lee, J. H.; Zheng, Q.; Du, X.; Zhang, X.; Xu, H.; Wang, Z.; Wu, Y.; Shen, X.; Cui, J.; Mai, Y.-W.; Kim, J.-K. SpiderWeb-Inspired Stretchable Graphene Woven Fabric for Highly Sensitive, Transparent, Wearable Strain Sensors. ACS Appl. Mater. Interfaces 2019, 11 (2), 2282-2294.

(22) Shi, G.; Zhao, Z.; Pai, J. H.; Lee, I.; Zhang, L.; Stevenson, C.; Ishara, K.; Zhang, R.; Zhu, H.; Ma, J. Highly Sensitive, Wearable,
Durable Strain Sensors and Stretchable Conductors Using Graphene/ Silicon Rubber Composites. Adv. Funct. Mater. 2016, 26 (42), 76147625.

(23) Zang, Y.; Zhang, F.; Huang, D.; Gao, X.; Di, C. A.; Zhu, D. Flexible Suspended Gate Organic Thin-Film Transistors for UltraSensitive Pressure Detection. Nat. Commun. 2015 DOI: 10.1038/ ncomms7269.

(24) Park, D. Y.; Joe, D. J.; Kim, D. H.; Park, H.; Han, J. H.; Jeong, C. K.; Park, H.; Park, J. G.; Joung, B.; Lee, K. J. Self-Powered RealTime Arterial Pulse Monitoring Using Ultrathin Epidermal Piezoelectric Sensors. Adv. Mater. 2017, 29 (37), 1-9.

(25) Nie, B.; Huang, R.; Yao, T.; Zhang, Y.; Miao, Y.; Liu, C.; Liu, J.; Chen, X. Textile-Based Wireless Pressure Sensor Array for HumanInteractive Sensing. Adv. Funct. Mater. 2019, 29 (22), 1808786.

(26) Lee, Y.; Park, J.; Cho, S.; Shin, Y. E.; Lee, H.; Kim, J.; Myoung, J.; Cho, S.; Kang, S.; Baig, C.et al.; et al. Flexible Ferroelectric Sensors with Ultrahigh Pressure Sensitivity and Linear Response over Exceptionally Broad Pressure Range. ACS Nano 2018, 12 (4), 4045.

(27) Park, J.; Lee, J.; Hong, J.; Ha, M.; Jung, Y.-D.; Lim, H.; Kim, S. Y.; Ko, Y. Giant Tunneling Piezoresistance of Composite Elastomers with Interlocked Microdome Arrays for Ultrasensitive and Multimodal. 2014, 5, 4689-4697 DOI: 10.1021/nn500441k.

(28) Qiu, Z.; Wan, Y.; Zhou, W.; Yang, J.; Yang, J.; Huang, J.; Zhang, J.; Liu, Q.; Huang, S.; Bai, N.et al. Ionic Skin with Biomimetic Dielectric Layer Templated from Calathea Zebrine Leaf. Adv. Funct. Mater. 2018, 28 (37), 1802343.

(29) Park, J.; Kim, J.; Hong, J.; Lee, H.; Lee, Y.; Cho, S.; Kim, S. W.; Kim, J. J.; Kim, S. Y.; Ko, H. Tailoring Force Sensitivity and Selectivity by Microstructure Engineering of Multidirectional Electronic Skins. NPG Asia Mater. 2018, 10, 163.

(30) Han, S.; Liu, C.; Huang, Z.; Zheng, J.; Xu, H.; Chu, S.; Wu, J.; Liu, C. High-Performance Pressure Sensors Based on 3D Microstructure Fabricated by a Facile Transfer Technology. Adv. Mater. Technol. 2019, 4 (5), 1800640.

(31) Zhou, J.; Xu, X.; Yu, H.; Lubineau, G. Deformable and Wearable Carbon Nanotube Microwire-Based Sensors for Ultrasensitive Monitoring of Strain, Pressure and Torsion. Nanoscale 2017, 9 (2), 604-612.

(32) Pan, L.; Chortos, A.; Yu, G.; Wang, Y.; Isaacson, S.; Allen, R.; Shi, Y.; Dauskardt, R.; Bao, Z. An Ultra-Sensitive Resistive Pressure Sensor Based on Hollow-Sphere Microstructure Induced Elasticity in Conducting Polymer Film. Nat. Commun. 2014 DOI: 10.1038/ ncomms4002.

(33) Sheng, L.; Liang, Y.; Jiang, L.; Wang, Q.; Wei, T.; Qu, L.; Fan, Z. Bubble-Decorated Honeycomb-Like Graphene Film as Ultrahigh Sensitivity Pressure Sensors. Adv. Funct. Mater. 2015, 25, 6545.

(34) Huang, Y.; Fan, X.; Chen, S. C.; Zhao, N. Emerging Technologies of Flexible Pressure Sensors: Materials, Modeling, Devices, and Manufacturing. Adv. Funct. Mater. 2019, 29 (12), 1-24.

(35) Wang, B.; Facchetti, A. Mechanically Flexible Conductors for Stretchable and Wearable E-Skin and E-Textile Devices. Adv. Mater. 2019, 31 (28), 1901408.

(36) Shi, H.; Al-Rubaiai, M.; Holbrook, C. M.; Miao, J.; Pinto, T.; Wang, C.; Tan, X. Screen-Printed Soft Capacitive Sensors for Spatial Mapping of Both Positive and Negative Pressures. Adv. Funct. Mater. 2019, 29 (23), 1809116.

(37) Li, R.; Si, Y.; Zhu, Z.; Guo, Y.; Zhang, Y.; Pan, N.; Sun, G.; Pan, T. Supercapacitive Iontronic Nanofabric Sensing. Adv. Mater. 2017, 29 (36), 1700253.

(38) Li, S.; Pan, N.; Zhu, Z.; Li, R.; Li, B.; Chu, J.; Li, G.; Chang, Y.; Pan, T. All-in-One Iontronic Sensing Paper. Adv. Funct. Mater. 2019, 29 (11), 1807343.

(39) Yang, J. C.; Mun, J.; Kwon, S. Y.; Park, S.; Bao, Z.; Park, S. Electronic Skin: Recent Progress and Future Prospects for SkinAttachable Devices for Health Monitoring, Robotics, and Prosthetics. Adv. Mater. 2019, 31 (48), 1904765.

(40) Roh, E.; Lee, H. B.; Kim, D. Il; Lee, N. E. A SolutionProcessable, Omnidirectionally Stretchable, and High-PressureSensitive Piezoresistive Device. Adv. Mater. 2017, 29 (42), 1703004. 
(41) Rogers, J. A.; Someya, T.; Huang, Y. Materials and Mechanics for Stretchable Electronics. Science 2010, 327, 1603.

(42) Kim, S.; Amjadi, M.; Lee, T. I.; Jeong, Y.; Kwon, D.; Kim, M. S.; Kim, K.; Kim, T. S.; Oh, Y. S.; Park, I. Wearable, Ultrawide-Range, and Bending-Insensitive Pressure Sensor Based on Carbon Nanotube Network-Coated Porous Elastomer Sponges for Human Interface and Healthcare Devices. ACS Appl. Mater. Interfaces 2019, 11, 23639.

(43) Yang, J. C.; Kim, J. O.; Oh, J.; Kwon, S. Y.; Sim, J. Y.; Kim, D. W.; Choi, H. B.; Park, S. Microstructured Porous Pyramid-Based Ultrahigh Sensitive Pressure Sensor Insensitive to Strain and Temperature. ACS Appl. Mater. Interfaces 2019, 11, 19472.

(44) Yoo, J. Y.; Seo, M. H.; Lee, J. S.; Choi, K. W.; Jo, M. S.; Yoon, J. B. Industrial Grade, Bending-Insensitive, Transparent Nanoforce Touch Sensor via Enhanced Percolation Effect in a Hierarchical Nanocomposite Film. Adv. Funct. Mater. 2018, 28 (42), 1804721.

(45) Liu, X.; Liu, D.; Lee, J. H.; Zheng, Q.; Du, X.; Zhang, X.; Xu, H.; Wang, Z.; Wu, Y.; Shen, X.; Cui, J.; Mai, Y.-W.; Kim, J.-K. SpiderWeb-Inspired Stretchable Graphene Woven Fabric for Highly Sensitive, Transparent, Wearable Strain Sensors. ACS Appl. Mater. Interfaces 2019, 11, 2282.

(46) Lai, Y. C.; Ye, B. W.; Lu, C. F.; Chen, C. T.; Jao, M. H.; Su, W. F.; Hung, W. Y.; Lin, T. Y.; Chen, Y. F. Extraordinarily Sensitive and Low-Voltage Operational Cloth-Based Electronic Skin for Wearable Sensing and Multifunctional Integration Uses: A Tactile-Induced Insulating-to-Conducting Transition. Adv. Funct. Mater. 2016, 26 (8), $1286-1295$.

(47) de Mulatier, S.; Nasreldin, M.; Delattre, R.; Ramuz, M.; Djenizian, T. Electronic Circuits Integration in Textiles for Data Processing in Wearable Technologies. Adv. Mater. Technol. 2018, 3 (10), 1700320.

(48) Huang, M.; Tunnicliffe, L. B.; Zhuang, J.; Ren, W.; Yan, H.; Busfield, J. J. C. Strain-Dependent Dielectric Behavior of Carbon Black Reinforced Natural Rubber. Macromolecules 2016, 49, 2339.

(49) Pan, F.; Chen, S. M.; Li, Y.; Tao, Z.; Ye, J.; Ni, K.; Yu, H.; Xiang, B.; Ren, Y.; Qin, F. 3D Graphene Films Enable Simultaneously High Sensitivity and Large Stretchability for Strain Sensors. Adv. Funct. Mater. 2018, 28 (40), 1-10.

(50) Luo, N.; Huang, Y.; Liu, J.; Chen, S. C.; Wong, C. P.; Zhao, N. Hollow-Structured Graphene-Silicone-Composite-Based Piezoresistive Sensors: Decoupled Property Tuning and Bending Reliability. Adv. Mater. 2017, 29 (40), 1-9.

(51) Wan, Y.; Qiu, Z.; Huang, J.; Yang, J.; Wang, Q.; Lu, P.; Yang, J.; Zhang, J.; Huang, S.; Wu, Z.; Guo, C. F. Natural Plant Materials as Dielectric Layer for Highly Sensitive Flexible Electronic Skin. Small 2018, 14 (35), 1-8.

(52) Tolvanen, J.; Hannu, J.; Jantunen, H. Hybrid Foam Pressure Sensor Utilising Piezoresistive and Capacitive Sensing Mechanisms. IEEE Sens. J. 2017, 17 (15), 4735-4746.

(53) Atalay, A.; Sanchez, V.; Atalay, O.; Vogt, D. M.; Haufe, F.; Wood, R. J.; Walsh, C. J. Batch Fabrication of Customizable SiliconeTextile Composite Capacitive Strain Sensors for Human Motion Tracking. Adv. Mater. Technol. 2017, 2 (9), 1700136.

(54) Kordas, K.; Pitkänen, O. Piezoresistive Carbon Foams in Sensing Applications. Front. Mater. 2019 DOI: 10.3389/ fmats.2019.00093. 